\title{
Identification of Protein Arginine Methyltransferase 5 as a Regulator for Encystation of Acanthamoeba
}

\author{
Eun-Kyung Moon', Yeonchul Hong², Dong-II Chung2 , Youn-Kyoung Goo², Hyun-Hee Kong,3,* \\ ${ }^{1}$ Department of Medical Zoology, Kyung Hee University School of Medicine, Seoul 02447, Korea; ${ }^{2}$ Department of Parasitology and Tropical \\ Medicine, Kyungpook National University School of Medicine, Daegu 41944, Korea; ${ }^{3}$ Department of Parasitology, Dong-A University College of \\ Medicine, Busan 49201, Korea
}

\begin{abstract}
Encystation is an essential process for Acanthamoeba survival under nutrient-limiting conditions and exposure to drugs. The expression of several genes has been observed to increase or decrease during encystation. Epigenetic processes involved in regulation of gene expression have been shown to play a role in several pathogenic parasites. In the present study, we identified the protein arginine methyltransferase 5 (PRMT5), a known epigenetic regulator, in Acanthamoeba castellanii. PRMT5 of $A$. castellanii (AcPRMT5) contained domains found in S-adenosylmethionine-dependent methyltransferases and in PRMT5 arginine-N-methyltransferase. Expression levels of AcPRMT5 were increased during encystation of $A$. castellanii. The EGFP-PRMT5 fusion protein was mainly localized in the nucleus of trophozoites. $A$. castellanii transfected with siRNA designed against AcPRMT5 failed to form mature cysts. The findings of this study lead to a better understanding of epigenetic mechanisms behind the regulation of encystation in cyst-forming pathogenic protozoa.
\end{abstract}

Key words: Acanthamoeba, encystation, cellulose synthase, endocyst

\section{INTRODUCTION}

Acanthamoeba is an opportunistic protozoan pathogen that can cause granulomatous encephalitis and keratitis [1]. Under harsh conditions or chemotherapeutic drugs, Acanthamoeba transforms into a resistant cyst form, causing significant problems in the treatment of Acanthamoeba infections [2]. Recently, several encystation-mediating factors have been found to inhibit the encystation of Acanthamoeba [3-9]. However, the regulation mechanisms of encystation mediating factors are, to our knowledge, still unknown.

Epigenetics studies the molecular mechanisms that control gene expression and is currently one of the most important fields in science. Epigenetics refers to heritable changes in gene expression that do not involve a change to the DNA sequence [10]. In higher eukaryotes, the study of epigenetic mechanisms responsible for various gene expression patterns has been widely used. Epigenetics is associated to processes such as gene and microRNA expression, DNA-protein interactions, suppression

- Received 14 September 2015, revised 10 December 2015, accepted 29 December 2015.

*Corresponding author (hhkong@dau.ac.kr)

(c) 2016, Korean Society for Parasitology and Tropical Medicine

This is an Open Access article distributed under the terms of the Creative Commons Attribution Non-Commercial License (http://creativecommons.org/licenses/by-nc/3.0) which permits unrestricted non-commercial use, distribution, and reproduction in any

medium, provided the original work is properly cited. of transposable element mobility, cellular differentiation, embryogenesis, X-chromosome inactivation, and genomic imprinting [11]. Epigenetic modifications can be grouped into 3 main categories: DNA methylation, histone modifications, and nucleosome positioning [11].

In yeast and humans, the posttranslational methylation of arginine residues in proteins affects myriad cellular processes, including transcription, RNA processing, DNA replication and repair, and signal transduction [12]. The protein arginine methyltransferase (PRMT) has been reported to catalyze these reactions. PRMTs have been identified in animals, fungi, plants, and protozoa. In parasitic protozoa, PRMTs have been identified in Trichomonas vaginalis, Entamoeba histolytica, Trypanosoma brucei, Plasmodium falciparum, and Toxoplasma gondii [12]. In addition to PRMT1 and PRMT5, cytosine methylated regions and 5-cytosine DNA methyltransferase (Ehmeth) have been characterized in E. histolytica [13]. However, to our knowledge, the presence of epigenetic regulation in Acanthamoeba has not been reported yet.

In order to understand epigenetic regulation in Acanthamoe$b a$, we analyzed the cDNA library of Acanthamoeba castellanii, which contains several genes with a methyltransferase domain. Among these sets, the homologue gene of PRMT5 (GenBank accession no. KT345169) was found. In this study, we demonstrate the regulatory role of protein arginine methyltransferase 
5 (PRMT5) during encystation in A. castellanii.

\section{MATERIALS AND METHODS}

\section{Amoeba cultivation and encystation}

A. castellanii Castellani was obtained from the American Type Culture Collection (ATCC 30011). A. castellanii trophozoites were axenically cultured in PYG medium that contains proteose peptone $0.75 \%(\mathrm{w} / \mathrm{v})$, yeast extract $0.75 \%(\mathrm{w} / \mathrm{v})$, and glucose $1.5 \%$ $(\mathrm{w} / \mathrm{v})$ at $25^{\circ} \mathrm{C}$ in a Sanyo incubator (San Diego, California, USA). A. castellanii encystation was induced in an encystation media $\left(0.1 \mathrm{M} \mathrm{KCl}, 0.008 \mathrm{M} \mathrm{MgSO}_{4}, 0.0004 \mathrm{M} \mathrm{CaCl}_{2}\right.$, and $0.02 \mathrm{M}$ 2-amino-2-methyl-1,3-propanediol, $\mathrm{pH}$ 9.0) for 3 days [14]. Mature cysts were counted under a light microscope after treating them with $0.5 \%$ SDS, and encystation ratios were calculated [15].

\section{Gene expression analysis based on real-time PCR}

Total RNA was purified using TRIzol reagent (Gibco BRL, Rockville, Maryland, USA), and CDNA synthesis was conducted using a RevertAid ${ }^{\mathrm{TM}}$ First Strand cDNA Synthesis Kit (Fermentas, Hanover, Indiana, USA). Real-time PCR was performed using the ABI PRISM ${ }^{\circledR} 7000$ sequence detection system (Applied Biosystems, Foster City, California, USA), using the default thermocycler program for sense and antisense primers (sense 5'-TTGACTACTCGGCTCTTCTGCC and antisense 5'TCGATGTCTTTCACCAGCAGG for protein arginine methyltransferase 5). All reaction mixtures used Sybr Premix Ex Taq (Takara, Otsu, Shiga, Japan). The $18 \mathrm{~S}$ ribosomal DNA was used as the reference gene [7]. Real-time PCR was performed to determine relative gene expression using the $2^{\Delta \Delta C T}$ method [16], and experiments were performed in triplicate.

\section{Stable transfection}

To investigate the intracellular localization of protein arginine methyltransferase 5, the gene was cloned into the pGAPDH vector using enhanced green fluorescent protein (EGFP) as a marker. This plasmid was transfected into live cells of $A$. castellanii. Following a previously described method, transient transfection was performed using the SuperFect transfection reagent (Qiagen, Hilden, Germany) [17]. Transfected cells were transferred to $50 \mu \mathrm{g}$ neomycin-containing G418 media and grown over several passages.

\section{Confocal microscopy}

The LSM 5 EXCITER Scalable confocal system (ZEISS, Ham- burg, Germany) was used to identify and observe amoebae expressing EGFP. EGFP- or DAPI (4',6-diamidino-2-phenylindole)mediated fluorescence was performed using bandpass filters covering excitation and emission wavelengths of 500 to 530 $\mathrm{nm}$ and 360 to $460 \mathrm{~nm}$, respectively.

\section{Gene silencing}

Small interfering RNA (siRNA) targeting protein arginine methyltransferase 5 of A. castellanii (AcPRMT5) was synthesized by Sigma-Proligo (Boulder, Colorado, USA), based on its cDNA sequence. The siRNA duplex with sense (5'-CAGCAAUUGCGCAGUAUCAdTdT) and anti-sense (5'-UGAUACUGCGCAAUUGCUGdTdT), designed against AcPRMT5, were used. The siRNA $(4 \mu \mathrm{g})$ was transfected into A. castellanii trophozoites at a cell density of $4 \times 10^{5}$ per well, as previously described [17].

\section{Statistical analysis}

Data are expressed as mean \pm SEM. Statistical significance was analyzed using an unpaired Student's $t$-test. A $P$-value of $<0.01$ was interpreted as statistically significant.

\section{RESULTS}

\section{Identification of protein arginine methyltransferase 5}

To identify the encystation-mediating factor of A. castellanii, we used the basic local alignment search tool (BLAST) to screen the cDNA library and identified a full-length open reading frame of the protein arginine methyltransferase 5 (AcPRMT5) (GenBank no. KT345169). AcPRMT5 contains domains found in S-adenosylmethionine-dependent methyltransferases (79148 amino acids) and in PRMT5 arginine-N-methyltransferase (29-281 amino acids). Amino acid sequence alignment of AcPRMT5 with the PRMT5 of Plasmodium reichenowi, Plasmodium falciparum, Dictyostelium discoideum, Entamoeba histolytica, Trichomonas vaginalis, and Trypanosoma brucei showed sequence similarity in the methyltransferase motifs I, post I, II, and III (Fig. 1).

To compare the mRNA expression levels of AcPRMT5 during encystation, we conducted real time-PCR. The expression level of AcPRMT5 was highly increased $72 \mathrm{hr}$ after the encystation induction (Fig. 2).

Intracellular localization of AcPRMT5 during encystation

A. castellanii EGFP-PRMT5 fusion protein showed a weak expression in the cytoplasm but a strong expression in the nucle- 


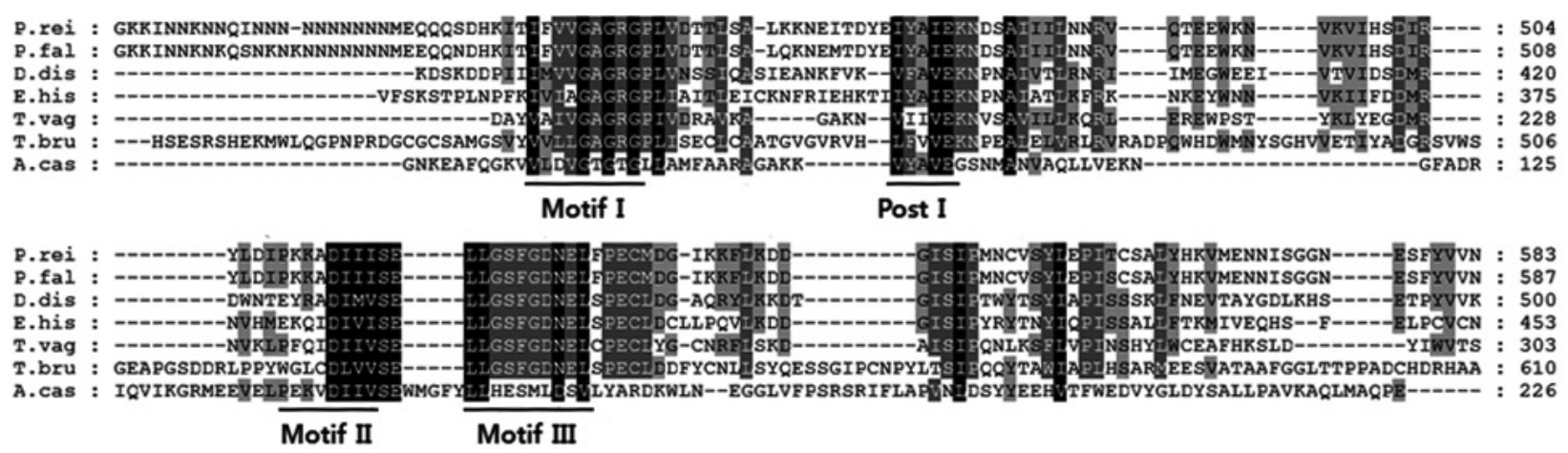

Fig. 1. Amino acid sequence alignment of Acanthamoeba castellanii PRMT5 with PRMT5 of Plasmodium reichenowi, Plasmodium falciparum, Dictyostelium discoideum, Entamoeba histolytica, Trichomonas vaginalis, and Trypanosoma brucei. Sequences characteristic of methyltransferase motifs I, post I, II, and III are underlined.

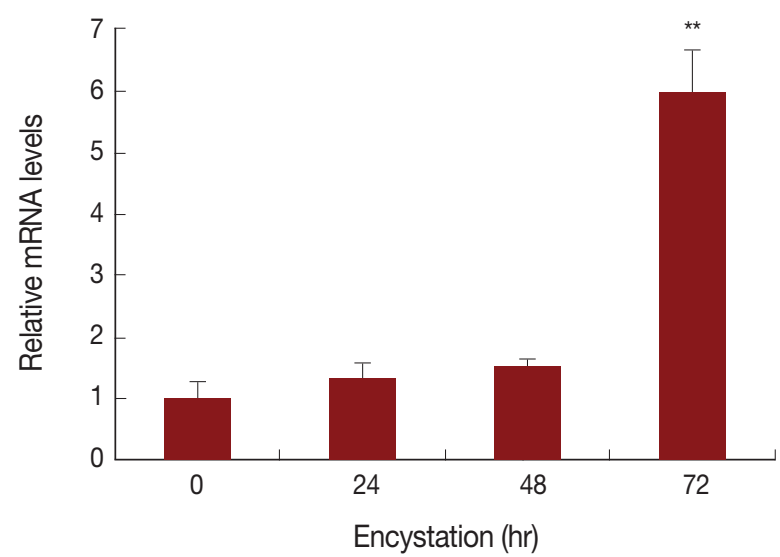

Fig. 2. mRNA expression of AcPRMT5 using real-time PCR analysis. AcPRMT5 showed high expression $72 \mathrm{hr}$ after encystation induction. Values indicate the mean $( \pm S D)$ of 3 experiments. ${ }^{\star *}$ Means are significantly different one another (Student's $t$-test, $P<0.01)$.

us of trophozoites (Fig. 3A). During encystation, EGFP-PRMT5 fusion protein showed a similar localization pattern, with most of the protein localized in the nucleus of cysts (Fig. 3B). These results were confirmed using DAPI staining.

\section{Gene silencing of AcPRMT5 by siRNA}

To determine the effect of PRMT5 during A. castellanii encystation, gene silencing by siRNA was performed. In the siRNA transfected A. castellanii, PRMT5 did not have a high expression level during encystation (Fig. 4A). Furthermore, after 72 hr of encystation induction, the formation of mature cysts was inhibited (20.4\%) (Fig. 4B).

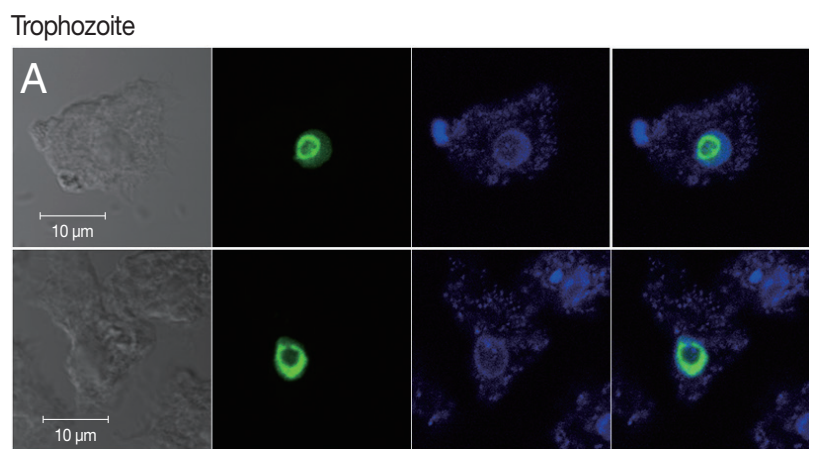

Cyst (encystation-72 hr)

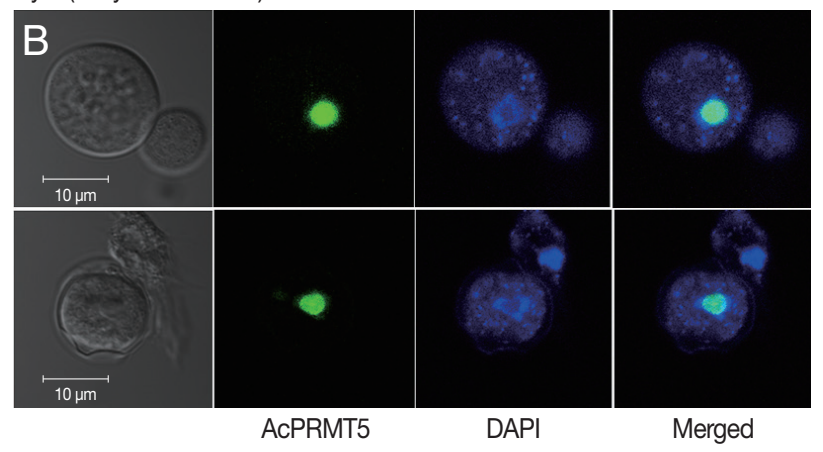

Fig. 3. Stable transfection of pGAPDHgPRMT5 for intracellular localization. (A) EGFP-PRMT5 fusion protein of $A$. castellanii was mainly localized in the nucleus of trophozoites. (B) After encystation, EGFP-PRMT5 fusion protein was localized in the nucleus of cysts. Localization of AcPRMT5 in the nucleus was confirmed by DAPI staining.

\section{DISCUSSION}

Post-translational modification of proteins by arginine methylation is involved in a variety of cellular processes including transcriptional regulation [18]. Protein arginine methyltransferases (PRMTs) transfer methyl groups from S-adenosylme- 
A

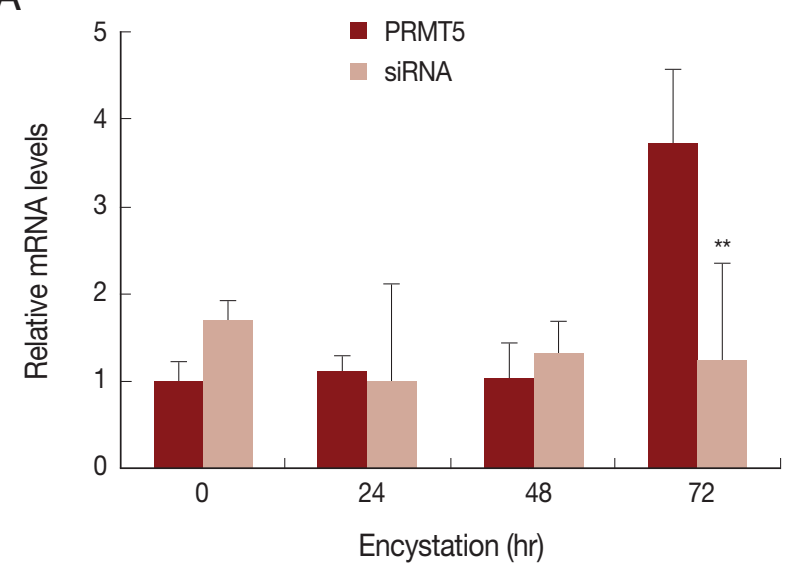

B

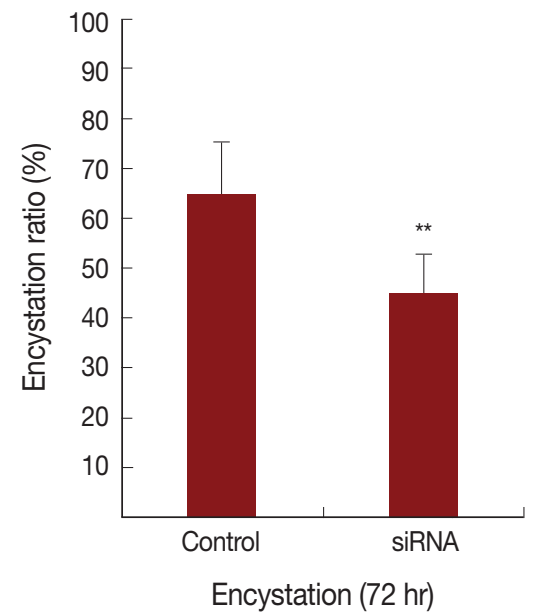

Fig. 4. Transfection of $A$. castellanii with siRNA designed against PRMT5. (A) PRMT5 showed a high expression level during encystation (घ), but was down-regulated in PRMT5-siRNA transfected cells $(\square)$. (B) This inhibition by PRMT5-siRNA was found to reduce encystation ratios. Values indicate the mean $( \pm \mathrm{SD})$ of 3 experiments. ${ }^{*}$ Means are significantly different one another (Student's $t$-test, $\left.P<0.01\right)$.

thionine (AdoMet) to arginine residues resulting in methylated arginines [19]. Three regions of sequence similarity, motif I, II, III, and post I have been reported in several AdoMet-dependent methyltransferases [20,21]. Methyltransferase motif I comprises 9 residues with the consensus sequence $(\mathrm{V} / \mathrm{L} / \mathrm{L})(\mathrm{L} / \mathrm{V})(\mathrm{D} /$ $\mathrm{E})(\mathrm{V} / \mathrm{I}) \mathrm{G}(\mathrm{G} / \mathrm{C}) \mathrm{G}(\mathrm{T} / \mathrm{P}) \mathrm{G}$, motif II comprises 8 residues with the consensus sequence $(\mathrm{P} / \mathrm{G})(\mathrm{Q} / \mathrm{T})(\mathrm{F} / \mathrm{Y} / \mathrm{A}) \mathrm{DA}(\mathrm{I} / \mathrm{V} / \mathrm{Y})(\mathrm{F} / \mathrm{I})(\mathrm{C} /$ $\mathrm{V} / \mathrm{L})$, and motif III comprises 10 conserved residues $\mathrm{LL}(\mathrm{R} / \mathrm{K})$ PGG(R/I/L)(L/I)(L/F/I/V)(I/L) after the end of motif II. The high degeneracy and narrow width of the post I motif did not allow its direct identification [20]. Post I was, therefore, appended to the description of motif I, considering 2 spacings: motif I-(10-30)-post I and motif I-(5-25)-post I. It was shown that post I comprised 5 residues with the consensus sequence $(\mathrm{V} / \mathrm{I} /$ L/F/Y/M/H)(I/V/L/F/Y/T/K/Q/D)(G/A/S/I/D)(V/I/L/N/S/F/C/ A) $(\mathrm{D} / \mathrm{E} / \mathrm{N})[20]$. The AcPRMT5 identified in this study showed high similarity to motif I, post I, and motif II (Fig. 1). Motif I is involved in AdoMet binding [20].

Many known arginine methyltransferases are proteins that have the ability to shuttle between the nucleus and the cytoplasm $[22,23]$. In mammalian cells, PRMT5 is localized in both the cytoplasm and the nucleus, where it is involved in the methylation of multiple histone and non-histone proteins [24]. Most of the AcPRMT5 was localized in the nucleus of trophozoites and cysts, while not much was found in the cytoplasm (Fig. 3). We hypothesize that AcPRMT5 regulates the transcriptional levels of encystation-associated genes by histone modification in the nucleus.

AdoMet-dependent PRMTs are generally classified into type
I and type II enzymes. PRMT1 is the predominant member of type I PRMTs, whereas PRMT5 is a representative member of type II PRMTs. PRMT1-mediated dimethylation of arginine 3 of histone 4 (H4R3) is associated with gene activation [25], while the methylation of H4R3 by PRMT5 is associated with gene repression [26]. The target substrate of AcPRMT5 has not been identified yet. However, the inhibition of encystation by AcPRMT5-siRNA transfection (Fig. 4) suggests that AcPRMT5 regulates encystation-mediating factors in A. castellanii. To our knowledge, no PRMT substrates have been identified in Entamoeba spp., nor have any functional studies been performed. Recently, PRMT1a of E. histolytica was reported to be associated with gene expression regulation by dimethylation of H4R8 [27]. T. gondii CARM1 (PRMT homologue) resulted in decreased parasite viability due to the transient expression of enzymedead mutant TgCARM1 in tachyzoites [28]. RNAi-mediated knockdown of T. brucei PRMT1 resulted in a slow growth phenotype and abnormal cell cycle progression [29]. In rat liver cells, PRMT5 negatively affected cyclin E1 promoter activity and cellular proliferation [30]. It was suggested that cyclin E1 is a key mediator of PRMT5-dependent cell cycle arrest [30]. In A. castellanii, we consider that PRMT5 is associated with cell cycle arrest. AcPRMT5 was highly expressed at a late stage of encystation (Fig. 2), and the inhibition of AcPRMT5 reduced the encystation ratio (Fig. 4B).

In this study, we described for the first time the role of protein arginine methyltransferase 5 as a regulator of encystation mediating factors in Acanthamoeba. Epigenetic regulators play an important role in control of gene expression by modifying 
the chromatin. Epigenetic factors are, therefore, important for development of many diseases. Further efforts should be made to understand how the epigenetic mechanisms of parasites may be used in therapeutic treatments.

\section{ACKNOWLEDGMENT}

This research was supported by the Basic Science Research Program of the National Research Foundation of Korea (NRF) funded by the Ministry of Education (grant no. 2014R1A1A 2058405).

\section{CONFLICT OF INTEREST}

We have no conflict of interest related to this work.

\section{REFERENCES}

1. Marciano-Cabral F, Cabral G. Acanthamoeba spp. as agents of disease in humans. Clin Microbiol Rev 2003; 16: 273-307.

2. Coulon C, Collignon A, McDonnell G, Thomas V. Resistance of Acanthamoeba cysts to disinfection treatments used in health care settings. J Clin Microbiol 2010; 48: 2689-2697.

3. Moon EK, Chung DI, Hong YC, Kong HH. Autophagy protein 8 mediating autophagosome in encysting Acanthamoeba. Mol Biochem Parasitol 2009; 168: 43-48.

4. Song SM, Han BI, Moon EK, Lee YR, Yu HS, Jha BK, Danne DB, Kong HH, Chung DI, Hong Y. Autophagy protein 16-mediated autophagy is required for the encystation of Acanthamoeba castellanii. Mol Biochem Parasitol 2012; 183: 158-165.

5. Leitsch D, Köhsler M, Marchetti-Deschmann M, Deutsch A, Allmaier G, Duchêne M, Walochnik J. Major role for cysteine proteases during the early phase of Acanthamoeba castellanii encystment. Eukaryot Cell 2010; 9: 611-618.

6. Moon EK, Chung DI, Hong YC, Kong HH. Characterization of a serine proteinase mediating encystation of Acanthamoeba. Eukaryot Cell 2008; 7: 1513-1517.

7. Moon EK, Hong YC, Chung DI, Kong HH. Cysteine protease involving in autophagosomal degradation of mitochondria during encystation of Acanthamoeba. Mol Biochem Parasitol 2012; 185: $121-126$

8. Lee JY, Song SM, Moon EK, Lee YR, Jha BK, Danne DB, Cha HJ, Yu HS, Kong HH, Chung DI, Hong Y. Cysteine protease inhibitor (AcStefin) is required for complete cyst formation of Acanthamoeba. Eukaryot Cell 2013; 12: 567-574.

9. Lee YR, Na BK, Moon EK, Song SM, Joo SY, Kong HH, Goo YK, Chung DI, Hong YC. Essential role for an M17 leucine aminopeptidase in encystation of Acanthamoeba castellanii. PLoS One 2015; 10: e0129884.

10. Jaenisch R, Bird A. Epigenetic regulation of gene expression: how the genome integrates intrinsic and environmental signals. Nat Genet 2003; 33: 245-254.

11. Portela A, Esteller M. Epigenetic modifications and human disease. Nat Biotechnol 2010; 28: 1057-1068.

12. Fisk JC, Read LK. Protein arginine methylation in parasitic protozoa. Eukaryot Cell 2011; 10: 1013-1022.

13. Fisher O, Siman-Tov R, Ankri S. Characterization of cytosine methylated regions and 5-cytosine DNA methyltransferase (Ehmeth) in the protozoan parasite Entamoeba histolytica. Nucleic Acids Res 2004; 32: 287-297.

14. Bowers B, Korn ED. The fine structure of Acanthamoeba castellanii (Neff strain). II. Encystment. J Cell Biol 1969; 41: 786-805.

15. Aqeel Y, Siddiqui R, Khan NA. Silencing of xylose isomerase and cellulose synthase by siRNA inhibits encystation in Acanthamoeba castellanii. Parasitol Res 2013; 112: 1221-1227.

16. Livak KJ, Schmittgen TD. Analysis of relative gene expression data using real-time quantitative PCR and the 2(-Delta Delta C(T)) method. Methods 2001; 25: 402-408.

17. Kong HH, Pollard TD. Intracellular localization and dynamics of myosin-II and myosin-IC in live Acanthamoeba by transient transfection of EGFP fusion proteins. J Cell Sci 2002; 115: 49935002.

18. McBride AE, Silver PA. State of the arg: protein methylation at arginine comes of age. Cell 2001; 106: 5-8.

19. Ghosh SK, Paik WK, Kim S. Purification and molecular identification of two protein methylases I from calf brain. Myelin basic protein- and histone-specific enzyme. J Biol Chem 1988; 263: 19024-19033.

20. Kagan RM, Clarke S. Widespread occurrence of three sequence motifs in diverse S-adenosylmethionine-dependent methyltransferases suggests a common structure for these enzymes. Arch Biochem Biophys 1994; 310: 417-427.

21. Katz JE, Dlakić M, Clarke S. Automated identification of putative methyltransferases from genomic open reading frames. Mol Cell Proteomics 2003; 2: 525-540.

22. Lee MS, Henry M, Silver PA. A protein that shuttles between the nucleus and the cytoplasm is an important mediator of RNA export. Genes Dev 1996; 10: 1233-1246.

23. Kessler MM, Henry MF, Shen E, Zhao J, Gross S, Silver PA, Moore CL. Hrp1, a sequence-specific RNA-binding protein that shuttles between the nucleus and the cytoplasm, is required for mRNA 3'-end formation in yeast. Genes Dev 1997; 11: 2545-2556.

24. Bedford MT, Clarke SG. Protein arginine methylation in mammals: who, what, and why. Mol Cell 2009; 33: 1-13.

25. Wang H, Huang ZQ, Xia L, Feng Q, Erdjument-Bromage H, Strahl $\mathrm{BD}$, Briggs SD, Allis CD, Wong J, Tempst P, Zhang Y. Methylation of histone $\mathrm{H} 4$ at arginine 3 facilitating transcriptional activation by nuclear hormone receptor. Science 2001; 293: 853-857.

26. Pal S, Vishwanath SN, Erdjument-Bromage H, Tempst P, Sif S. Human SWI/SNF-associated PRMT5 methylates histone H3 arginine 8 and negatively regulates expression of ST7 and NM23 tumor suppressor genes. Mol Cell Biol 2004; 24: 9630-9645.

27. Borbolla-Vázquez J, Orozco E, Betanzos A, Rodríguez MA. Ent- 
amoeba histolytica: protein arginine transferase 1a methylates arginine residues and potentially modify the $\mathrm{H} 4$ histone. Parasit Vectors 2015; 8: 219.

28. Saksouk N, Bhatti MM, Kieffer S, Smith AT, Musset K, Garin J, Sullivan WJ Jr, Cesbron-Delauw MF, Hakimi MA. Histone-modifying complexes regulate gene expression pertinent to the differentiation of the protozoan parasite Toxoplasma gondii. Mol Cell Biol 2005; 25: 10301-10314.

29. Subramaniam C, Veazey P, Redmond S, Hayes-Sinclair J, Cham- bers E, Carrington M, Gull K, Matthews K, Horn D, Field MC. Chromosome-wide analysis of gene function by RNA interference in the African trypanosome. Eukaryot Cell 2006; 5: 15391549.

30. Fabbrizio E, El Messaoudi S, Polanowska J, Paul C, Cook JR, Lee JH, Negre V, Rousset M, Pestka S, Le Cam A, Sardet C. Negative regulation of transcription by the type II arginine methyltransferase PRMT5. EMBO Rep 2002; 3: 641-645. 